\title{
LA EDUCACIÓN EN GEOCIENCIAS COMO EJE DE FORMACIÓN CIUDADANA EN EL CONTEXTO ECUATORIANO
}

Diego Andrés Peña Arcos, Ph.D.

Doctor en Ciencias Geofísicas (Colombia). Docente Tiempo completo de la Facultad de Ingenierías de la Universidad Tecnológica ECOTEC (Ecuador). dpena@ecotec.edu.ec

Camilo Arias Ruiz, Ph.D.

Doctor en Ciencias de la Tierra y Medio Ambientales (Francia). Investigador Postdoctoral, Laboratoire de Planétologie et Géodynamique LPG-Nantes, Université de Nantes, Nantes, Francia. camilo.arias.ruiz@gmail.com

\section{ARTÍCULO DE REFLEXIÓN}

Recibido: 5 de octubre 2019.

Aceptado: 28 de febrero de 2020.

\section{RESUMEN}

Las Geociencias abarcan el conjunto de disciplinas científicas que construyen conocimientos sobre el planeta. Dadas sus características y objetos de estudio, es importante reflexionar acerca de sus contenidos para la educación básica en el contexto ecuatoriano, teniendo en cuenta que esta se basa en tres objetivos: la formación de conocimientos y competencias académicas, el desarrollo de habilidades para la vida laboral, y la formación integral ciudadana. A partir de esta formación, científica y ciudadana, los estudiantes deberán ser capaces de asumir responsabilidades ciudadanas y ser conscientes del impacto de sus acciones en el entorno, así como servir como fiscalizadores y generadores de proyectos relacionados con las problemáticas ambientales derivadas de intervenciones humanas en la naturaleza. En el presente trabajo, se analizó el currículo de los niveles de educación obligatoria del área de Ciencias Naturales para Educación General Básica (EGB) y Bachillerato General Unificado (BGU), de forma que se puedan relacionar los contenidos de Geociencias con la perspectiva de formación ciudadana. Finalmente se llega a algunas conclusiones a partir de las reflexiones iniciales, y se hacen algunas propuestas de modificación y consolidación de conocimientos en Geociencias que formen una consciencia ciudadana integral e interesada por la sociedad y su entorno medio ambiental. 
Palabras clave: educación, geociencias, ciudadanía, formación integral.

\section{ABSTRACT}

Geosciences cover the set of scientific disciplines that build knowledge about the planet. Given its characteristics and objects of study, it is important to reflect on its contents for basic education in the Ecuadorian context, bearing in mind that it is based on three objectives: the formation of academic knowledge and skills, the development of life skills labor, and comprehensive citizen training. From this scientific and citizen training, students should be able to assume citizen responsibilities and be aware of the impact of their actions on the environment, as well as serve as supervisors and generators of projects related to environmental problems derived from human interventions in nature. In this work, the curriculum of the levels of compulsory education in the area of Natural Sciences for Basic General Education (EGB) and Unified General Baccalaureate (BGU) was analyzed, so that the contents of Geosciences can be related to the perspective of training citizen. Finally, some conclusions are reached from the initial reflections, and some proposals are made for the modification and consolidation of knowledge in Geosciences that form an integral citizen consciousness interested in society and its environmental environment.

Keywords: education, geoscience, citizenship, comprehensive training.

\section{INTRODUCCIÓN}

En los últimos 50 años, la enseñanza en Geociencias se ha convertido en punto de encuentro de distintas ciencias y estudios que buscan comprender y reflexionar acerca de su rol dentro del desarrollo del mundo contemporáneo. Estas décadas han sido de continua transformación en la forma en la vemos y estudiamos el mundo, con nuevas escuelas, nuevos alumnos y necesidades, de forma tal que la manera tradicional de la enseñanza ya no es suficiente para un desarrollo integral y actualizado (Lopes y Macedo, 2011).

Las Geociencias, que equivalen a Ciencias de la Tierra, abarcan el conjunto de disciplinas científicas que construyen conocimientos sobre nuestro planeta, para comprender los complejos y variados procesos involucrados en su evolución, desde su origen hasta la actualidad. En pocas palabras, las Geociencias son las que permiten un mejor y más profundo entendimiento de nuestro entorno y su relación con el ser humano.

Es justamente en esta relación con el ser humano donde parte el papel decisivo de las Geociencias en la formación ciudadana. Todas las intervenciones humanas en el entorno 
tienen consecuencias y estas, de una u otra manera, están relacionadas con problemáticas geocientíficas como son: el Medio Ambiente, los Recursos (petróleo, minería, suelos, agua, etc.) y los Riesgos Naturales (inundaciones, avalanchas, sismos, vulcanismo, etc.). Los efectos de intervenciones humanas deficientes, de forma directa o indirecta, vulneran los derechos de la población a gozar de calidad de vida y de un ambiente saludable. Consecuentemente, se atenta contra el desarrollo sustentable que, en principio se entiende como la mejora de la calidad de vida de la sociedad y la preservación del equilibrio ecológico, promoviendo el crecimiento económico (Lacreu, 2015).

Dadas las características geográficas, geológicas, demográficas y ambientales del Ecuador, su territorio es altamente sensible a una variada gama de accidentes ambientales, originados o no, en la acción humana. Dichos accidentes aluden daños en la salud, la economía, las comunicaciones, en la cultura o el patrimonio relacionados con contaminación de aguas, suelos y aire, con derrumbes o deslizamientos en carreteras, minas o asentamientos precarios, las inundaciones y sequias, o con terremotos y la presencia de cenizas volcánicas en la atmósfera. De ahí que, en la educación básica ecuatoriana, estas temáticas no pueden estar ausentes y que los conocimientos impartidos (o compartidos), excedan la mera enunciación o descripción de los problemas. De hecho, es absolutamente necesario un conocimiento más profundo del entorno y sus problemas, a fin de mejorar la comprensión acerca de las causas, las consecuencias, intensidades y la probable evolución de estos procesos, creando así una conciencia ciudadana integral desde los primeros niveles de escolaridad.

\section{REVISIÓN TEÓRICA}

\section{Educación en Geociencias}

Se denomina Geociencias al conjunto de disciplinas científicas que estudian la estructura, morfología, evolución y dinámica del planeta Tierra. Su campo de acción va desde la comprensión de las causas que originan los fenómenos naturales, como afectan al ser humano y cómo el ser humano influye en la naturaleza con sus acciones. Las Geociencias permiten entender los procesos naturales que han favorecido y/o amenazado la vida de los seres humanos, y su estudio está ligado tanto al estudio de los flujos de energía en la naturaleza y al aprovechamiento de los mismos, como a la prevención de riesgos medioambientales, sísmicos, meteorológicos y volcánicos, entre otros. Este conjunto incluye disciplinas como la Geología, Geofísica, Oceanografía, Meteorología, Climatología, 
Paleontología, Edafología, Biología, Geoquímica, etc., las cuales se encargan de sistemas específicos con cuerpos de conocimientos con identidades propias. A pesar de esta diferenciación, es interesante percibir que existen conocimientos originados en estas áreas en la cotidianidad.

Por esto, la selección de contenidos geocientífico para educación básica ha estado sometida a tensiones entre profesionales de Geociencias y de Educación (Lacreu, 2015), los cuales difieren cuales serían los conocimientos necesarios para la formación de científicos y la de ciudadanos. Al dar una base geocientífica al cotidiano, se va construyendo una conciencia de que todo aquello que pasa al alrededor tiene una explicación científica, que todas nuestras acciones tienen consecuencias ambientales y que todos, sin excepción, tenemos un rol en la conservación de nuestro entorno. De igual manera, la UNESCO también afirma que, "una persona que no maneje una formación científica adecuada, tiene limitada su capacidad de participación democrática", dejando en evidencia el poder transformador y formador de ciudadanía de las Geociencias" (Macedo, 2016).

\section{Analfabetismo geocientífico}

En la vida cotidiana suele ignorarse que convivimos y dependemos de los productos obtenidos y/o elaborados a partir de los Recursos Naturales tanto Renovables como No Renovables y además se ignora quién los produce, dónde se encuentran, como se extraen y purifican, cómo y cuándo se formaron, qué cantidades disponemos, etc. Esa ignorancia se debe, en gran parte, a que se da mayor importancia a la transformación industrial de las materias primas, pero no sus fuentes genuinas. Dicha circunstancia, comprende lo que se denomina "analfabetismo geocientífico" que contribuye a consolidar ideas intuitivas erróneas como aquella de que las rocas y minerales presentes en el Planeta no se agotarán nunca y que se puede disponer eternamente de ellas en las cantidades que se necesitan. Este "analfabetismo geocientífico está relacionado también con la proliferación de "fake news" o noticias falsas acerca de eventos catastróficos de grande magnitud como la que circuló en redes sociales luego del terremoto de Pedernales del 16 de abril de 2016.

Es importante notar como todo lo anterior está relacionado con un déficit en la información que debió haberse dado en algún punto de la formación de la población. Fenómenos como las sequías, inundaciones o terremotos se manifiestan sorpresivamente, usualmente sin previo aviso y cobran gran cantidad de víctimas y daños materiales, que podrían ser minimizados mejorando el acceso a la información geocientífica. Por otro lado, esta 
percepción de agotamiento imposible, no provoca las mismas angustias y preocupaciones que derivan de estos fenómenos, pero que a largo plazo podrían cobrar tantas o hasta más víctimas que sismos o volcanes.

Esta es una temática que gana cada vez mayor popularidad en los medios, donde se habla continuamente de buenas administraciones ambientales, uso sustentable de recursos, conservación, del Medio Ambiente, etc., pero que la mayoría de veces carece de una buena conceptualización científica. Una propuesta interesante para mudar esta situación es considerar al Medio Ambiente como un recurso natural no renovable, reconociendo su evolución y relación con el ser humano. Primariamente, el Medio Ambiente, está definido por las características geológicas y climáticas de las distintas regiones (Lacreu, 1994), y posee un equilibrio que luego es modificado por las comunidades al instalar las explotaciones agrarias, mineras, los centros urbanos e industriales, etc. Dichas características primarias pueden identificarse con la disponibilidad de recursos tales como agua potable, rocas y minerales, suelos aptos para la producción agropecuaria, superficie y subsuelo estables para la fundación de edificios, etc. pero también se caracterizan por la acción potencial de riesgos geológicos tales como avalanchas, inundaciones, sismos o vulcanismo.

Una sociedad que emplea permanentemente recursos naturales, debe procurar que la formación general de los futuros ciudadanos incorpore de forma crítica las Geociencias. Los contenidos conceptuales, procedimentales y actitudinales involucrados en este campo interdisciplinario permiten enseñar y eventualmente aprender las razones de la distribución de los recursos naturales del país y del uso que de ellos se hace. Esta perspectiva, contribuirá a desarrollar una "cultura solidaria" para que los ciudadanos puedan defender el bien común representado por los Recursos Naturales No Renovables.

\section{Formación Ciudadana}

La definición y aplicación del concepto de formación ciudadana ha evolucionado a través de los años adaptándose a las condiciones de cada sociedad. En principio, la formación ciudadana se define como aquel tipo de educación que busca fortalecer los espacios de convivencia social entre las personas. Esto deriva en que cada individuo tiene una responsabilidad social sobre sus acciones y las que, de una u otra manera, afecten su vida. Pero esta responsabilidad social no es única, existiendo una gran diversidad de enfoques dependiendo de los objetivos y de los objetos de responsabilidad en juego. 
Quezada (2010) agrupó estos enfoques y determina, como factor de convergencia, al Enfoque Transformacional en el que describe la Formación como un ámbito cuyo objetivo principal es la formación de ciudadanos responsables. La formación ciudadana materializa uno de los aspectos esenciales del modo en que la Educación Básica debe jugar su papel como actor de la responsabilidad social.

De esta manera, el objetivo principal de la formación ciudadana es desarrollar sujetos conocedores de sus derechos, deberes, obligaciones y capacitados para la participación activa y responsable dentro del sistema democrático contribuyendo al desarrollo sustentable del país. De esta forma, la ciudadanía se considera como "el ejercicio de una práctica indefectiblemente política y fundamentada en valores como la libertad, la igualdad, la autonomía, el respeto a la diferencia, la solidaridad, la tolerancia y la desobediencia a poderes totalitarios" (Gentili, 2000 p.31).

Por otro lado, los ciudadanos no deben conformarse solo con cumplir las normas, sino que también deben controlar el efectivo cumplimiento de las mismas. Muchos de los problemas ambientales actuales no se originan por falta de leyes, normativas o protocolos que guíen las buenas prácticas, sino porque estas son incumplidas. Ya sea por negligencia o por omisión intencional, esto vulnera las garantías que aseguran las relaciones respetuosas entre los integrantes de una comunidad y la naturaleza, y el derecho a vivir con calidad de vida.

En Ecuador, la formación ciudadana se encuadra como eje transversal de la EGB y el BGU. Como parte de las políticas del estado ecuatoriano, el Consejo de Participación Ciudadana y Control Social (CPCCS) desarrolla programas de escuela de formación ciudadana disponibles para toda la población. El programa tiene por objetivo capacitar a la ciudadanía y generar procesos de empoderamiento y corresponsabilidad en los distintos asuntos de interés público. Se enmarca en el Eje 3 del Plan Nacional de Desarrollo 2017-2021 "Más Sociedad Mejor Estado", que incentiva una participación activa de la sociedad, que demanda un Estado con mejores capacidades de intervención y de trabajo articulado entre las instituciones gubernamentales y la población.

\section{La enseñanza de las Geociencias en la educación básica ecuatoriana}

A partir de septiembre del 2016, se establece la más reciente actualización del Currículo de los niveles de educación obligatoria, la cual indica cuales son los contenidos deben ser impartidos y que objetivos deben lograrse en las unidades de educación básica. Dentro de los 
contenidos básicos comunes para la EGB, las Geociencias están presentes en el área de Ciencias Naturales, en el Bloque 4: La Tierra y el Universo. En los subniveles Preparatoria y Elemental, los contenidos planeados están relacionados con la naturaleza y los recursos naturales, siendo sus títulos: "Características de los elementos naturales" y "Recursos Naturales: clasificación (renovables y no renovables)". Por otro lado, en los subniveles Medio y Superior, los contenidos están más relacionados con los procesos geológicos y dinámica de la Tierra, como: "La Tierra: estructura y fundamentos de la tectónica de placas", "Fenómenos geológicos y sus efectos", "Efectos de las erupciones volcánicas", "Procesos geológicos y extinciones masivas de la Tierra", Movimiento de placas tectónicas" y "Formación y ciclo de las rocas". En cada uno de ellos se detallan los contenidos básicos comunes (conceptuales, procedimentales y actitudinales), aspirando a que al finalizar la EGB se alcancen los siguientes logros:

- Aprecia el paisaje local desde la identificación de las características de los elementos naturales y construidos, el tiempo atmosférico sus cambios y efectos en los seres vivos.

- Establece las características, importancia y localización de los recursos naturales (renovables y no renovables) de las regiones del Ecuador y emite razones para realizar una explotación controlada.

- Analiza, desde la indagación de diversas fuentes, los efectos de los fenómenos geológicos sobre el planeta Tierra, tomando en cuenta la composición.

- Sistema Solar, la estructura de la Tierra, la influencia de las placas tectónicas en la formación de la cordillera de los Andes y la distribución de la biodiversidad en las regiones naturales del Ecuador, reforzando su análisis con las contribuciones científicas al campo de la vulcanología del país.

- Explica el fenómeno de movimiento de las placas tectónicas, partiendo de la relación con las erupciones volcánicas, la formación y ciclo de las rocas, infiriendo los efectos de estos procesos en los cambios climáticos y distribución de organismos en los ecosistemas.

Concluyendo, un estudiante de EGB será capaz de integrar los conceptos de las ciencias biológicas, químicas, físicas, geológicas y astronómicas, para comprender la ciencia, la 
tecnología y la sociedad, ligadas a la capacidad de inventar, innovar y dar soluciones a la crisis socioambiental (Ministerio de Educación, 2016).

En contraste, dentro de los contenidos básicos comunes en BGU, las Geociencias no se encuentran tan evidentes, siendo relegadas a ejemplos y casos de estudio de la Física o por limitados contenidos de astronomía básica.

\section{CONCLUSIONES}

El papel que juegan las geociencias en la formación ciudadana aún es motivo de debate entre profesionales y educadores. Dada la complejidad de sus contenidos y las posibilidades de formación más allá de la simple parte académica, es necesario abrir un amplio debate interdisciplinario a fin de definir las dimensiones científicas, pedagógicas y epistemológicas necesarias para encuadrar a la educación en Geociencias como eje de formación ciudadana.

Un análisis profundo de los contenidos del currículo de Ciencias Naturales en EGB y BGU muestra que, el principal déficit de contenidos geocientíficos enseñados se refiere a los recursos naturales: energía, minería, suelos y aguas. Dentro de los contenidos, los recursos naturales son presentados como productos, representados por bienes y territorios, que el ser humano dispone para su beneficio y desarrollo. Esto deriva en que la selección de contenidos a enseñar deje de fuera a los procesos generadores de recursos y riesgos naturales, dificultando la construcción de aprendizajes significativos y críticos sobre los recursos y conduce a entender al recurso como "riqueza" de una región (Chevallard, 1980; Lacreu, 1997).

Para ello, es útil la formulación de interrogantes políticos para cuyas respuestas sea necesario incorporar contenidos de las geociencias, por ejemplo: ¿En qué medida nuestra sociedad será capaz de compatibilizar la demanda de recursos mineros indispensables para su desarrollo con las escasas reservas y costo ambiental derivado de su explotación?; o ¿Cuáles son los motivos por los que gran parte de Estudios de Impacto Ambiental no han logrado evitar la continua degradación de suelos, pérdida de biodiversidad y la contaminación de aguas por accidentes o negligencia operativa?. Son este tipo de preguntas las que buscan generar esta cultura de transformación en la Educación básica ecuatoriana, formando sujetos con ciencia y consciencia, responsables y comprometidos con la vida democrática y ciudadana. Sujetos capaces de defender sus derechos, los del entorno y de la sociedad en la que habitan. 


\section{REFERENCIAS BIBLIOGRÁFICAS}

Gentili, P. (2000). Códigos para la ciudadanía. La formación ética como práctica de la libertad Ed. Santillana (pp. 143). Buenos Aires.

Lacreu, H. L. (2015). Geociencias para la formación ciudadana. In XIV Congreso Geológico Chileno. Actas (pp. 469-472).

Lacreu, H. L. (2009). La importancia de las geociencias para la construcción de ciudadanía en el currículo de la enseñanza básica en Processo Curricular, diferentes dimensões, Cap. 1:17-36, Florianópolis.

Lacreu, H. L. (1994). Ciencias Geológicas y Medio Ambiente. II Simp. Nac. Sobre la Ens. De la Ciencia y la Tecnología. SINECYT94-Julio/94, Bs. As.

López, A., y Macedo, E. (2011). Teorías de Currículo. São Paulo: Cortez Editoral.

Ministerio de Educación (2016). Currículo de los niveles de educación obligatoria. Ecuador.

Quezada, R. G. (2011). La responsabilidad social universitaria como desafío para la gestión estratégica de la Educación Superior: el caso de España The university social responsibility as a challenge to the strategic management of Higher Education: the case of Spain. Revista de educación, 355, (pp. 109-133). 